\title{
Does the electron have an anomalous electric dipole moment?
}

\author{
Engel Roza \\ Philips Research Labs, Eindhoven, The Netherlands (retired) \\ Email: engel.roza@onsbrabantnet.nl
}

\begin{abstract}
An analysis is presented of the possible existence of a second anomalous dipole moment of Dirac's particle next to the angular one. It includes a discussion why, in spite of his own derivation, Dirac has denied its relevancy. It is shown why since then it has been overlooked and why it has vanished from leading textbooks. A critical survey is given on the reasons of its reject, including the failure of attempts to measure and its perceived violation of the CPT theorem. Moreover, by reference from literature, the possible impact is discussed in the nuclear domain and in the gravitational domain if it would exist.
\end{abstract}

Keywords: anomalous electric dipole moment; Dirac particle; Pauli's spin vector; isospin

\section{Introduction}

In his classic paper on electrons, Paul Dirac has derived a basic 4-dimensional wave equation for an electron in motion subject to a vector potential $\boldsymbol{A}\left(A_{0}, A_{x}, A_{y}, A_{z}\right)$. In this equation [1, eq. 15/16], an anomalous electric dipole moment shows up, next to the well-known anomalous magnetic dipole moment. Dirac doubted whether it could have a physical interpretation, the more because it appeared in a quantity with an imaginary sign as compared with a similar expression for the magnetic dipole moment. Where a magnetic dipole moment makes sense as a manifestation of angular spin, a similar physical manifestation for an electric dipole moment is not obvious.

This is a first reason why, since then, the electric dipole moment of an electron has been ignored. The second reason is, that experimental attempts to reveal an electric dipole moment (EDM), if it would exist, all failed. Presently, the Particle Data Group (PDG) has set an upper limit for its value as [2],

$\mathrm{EDM}<0.8710^{-30} q \mathrm{~m}$,

where $q$ is the elementary charge.

The third reason why an electron dipole moment for an electron has been rejected is because of its perceived violation of the CPT parity [3].

There is somewhat more. It is quite curious that in the highly reputed textbook of Bjorken and Drell, the electric dipole moment is no longer mentioned. Bjorken and Drell have decomposed Dirac's four-component wave function $\psi\left(\psi_{1}, \psi_{2}, \psi_{3}, \psi_{4}\right)$ into two twocomponents wave equations for the non-relativistic domain, a dominant one $\psi\left(\psi_{1}, \psi_{2}\right)$ and 
a minor one $\chi\left(\chi_{1}, \chi_{2}\right)$. See [4, eq. 1.32 and 1.33]. Dirac's electric dipole moment no longer shows up, while the magnetic dipole moment is clearly present. One might guess that its disappearance is due to the non-relativistic restriction. In Griffiths textbook [5], the electrons's electric dipole moment is not mentioned.

Let us inspect all those arguments step by step. First of all, Dirac's doubt is not a proof. The imaginary signed term pointing to a possible existence of an electric dipole moment is just a term in a wave equation with complex quantities. It might well be that in a proper energetic interpretation of the wave equation, the term will contribute a real quantity. Furthermore, the difficulty of physical interpretation might be due to an unexpected property of an electron. Let us suppose that the electron, similarly like all physical particles, is subject to the Heisenberg uncertainty. Let us suppose, just by hypothesis, that its position $d$ in its center of mass frame can be explained as the result of a motion with ultra-relativistic speed near vacuum light velocity $c$, such that

$d=c \Delta t$

Applying Heisenberg's relationship $\Delta E \Delta t=\hbar / 2,[6]$, on (1), we get

$$
d=c \Delta t=c \frac{\hbar}{2} \frac{1}{\Delta E} \rightarrow d=c \frac{\hbar}{2} \frac{1}{m c^{2}}=c \frac{\hbar}{2} \frac{1}{m c^{2}} \rightarrow \mu_{m}=m d=\frac{\hbar}{2 c},
$$

where $\mu_{m}$ has the dimensions of a dipole moment, expressed in terms of Planck's reduced constant $\hbar$ and a virtual mass $m$ (not to be confused with the particle's rest mass $m_{0}$ ).

Hence, it might well be that Dirac's doubt might be fed by not considering the possibility of a Heisenberg type vibration as an elementary motion of a Dirac particle next to an elementary angular motion.

Let us proceed by discussing the failure of measurement. Now we have suggested, by argumentation, the possible existence of a mechanical vibration moment $\hbar / 2 c$ next to the mechanical angular moment $\hbar$, the question has to be addressed how to relate these mechanical motions with the hypothetical existence of an electric dipole moment and the existence of a magnetic dipole moment of an electron with its elementary charge $q$ and its mass $m_{0}$. The magnetic one is well known from textbooks as [5],

$$
\mu_{m}=q \frac{\hbar}{2 m_{0}} \approx\left(9.2710^{-24} \mathrm{C} \mathrm{m}^{2} \mathrm{~s}^{-1}\right)
$$

The electric one needs to be derived by theory as shown later in this text. It appears being,

$$
\mu_{e l}=\frac{\hbar}{2 c} \frac{q}{m_{0}}\left(\approx 3.0910^{-32} \mathrm{Cm}\right)
$$


This is quite different from the PDG value quoted before. The discrepancy is due to a basic difference between electric dipole moment EDM as defined in the context of PDG and the anomalous electric dipole moment $\mu_{e l}$ as meant by Dirac. The latter is a quantum mechanical phenomenon, while EDM is not. Instead, the EDM is a consequence of a presupposed spatial structure of an electron with some charge distribution. If the electron is pointlike indeed, there is no EDM. The anomalous one shows up as a quantum mechanical vector with eigenvalues, even if the particle is pointlike.

Let us now discuss the perceived CPT violation, thereby using the arguments quoted in [3]. Here, the interaction Hamiltonians, $H_{E}$ and $H_{M}$ for the electric dipole moment and the magnetic dipole moment are, respectively, expressed as,

$$
H_{E}=-d_{E} \mathbf{S} \cdot \mathbf{E} \quad \text { and } \quad H_{M}=-d_{M} \mathbf{S} \cdot \mathbf{B} \text {, }
$$

where $\mathbf{S}, \mathbf{E}, \mathbf{B}, d_{E}$ and $d_{M}$, respectively, are the spin vector, the electric field strength and the magnetic field strength, and where $d_{E}$ and $d_{M}$ are the strengths of the dipoles. The interaction takes place between external fields on the one hand and on the other hand the internal axial magnetic field aligned with the spin vector and the internal electric field set up between two poles. The violation is perceived on the consideration that, under parity reversal, both the spin vector $\mathbf{S}$ and the axial magnetic field $\mathbf{B}$ change sign, such that the magnetic dipole moment maintains its parity, while the electric dipole moment's parity is violated because the internal electric field does not change sign, while the spin vector does. The same holds for time reversal. The fallacy here is, that, where the magnetic dipole moment $(q \hbar / 2 m)$ is determined by a spin vector with eigen value $S=\hbar / 2$, the electric dipole moment ( $q \hbar / 2 \mathrm{~cm}$ ) is determined by an isospin vector with eigen value $S=\hbar / 2 c$. It will be clear that the dimension of these two vectors is different. Parity reversal and time reversal change signs similarly as in the case of the magnetic dipole moment.

After having discussed the three arguments physical interpretation, magnitude and parity violation, we are left with the problem why the electric dipole moment does not show up in the Bjorken-Drell $(2 \times 2)$ - wave function, while it does in Dirac's 4-component one. Eventually, it will be an Hamiltonian expression that determines its viability. Hence, we shall straightforwardly analyze the problem by an inspection of the Hamiltonian. This will reveal the actual crux of the problem. The approach that we shall adopt is meant to get some understanding of three puzzles. All three have to do with my difficulty to understand Dirac's result properly. The puzzles that I would like to address are,

1. Why does Dirac's result not show a full symmetry over the four space-time dimensions? A three component Pauli vector $\overline{\mathrm{O}}=\overline{\mathrm{O}}\left(\mathrm{o}_{1}, \mathrm{O}_{2}, \mathrm{O}_{3}\right)$ is mixed up in in-products with, respectively, an electric field and a magnetic field. Hence, over three dimensions. A full symmetry would show a mix up of a four component Pauli-type vector with the four components of a vector potential. Hence, the question is whether, under the separation of the $p_{0}$ component from 
the $p_{i}$ components in the $4 \mathrm{D}$ momentum vector $\boldsymbol{p}=\boldsymbol{p}\left(p_{0}, p_{x}, p_{y}, p_{z}\right)$, the full general relativistic character has been maintained.

2. It is not immediately obvious why the interaction terms of Pauli's vector with the vector potential would result in the rather simple energetic interpretation as quoted in (5). In (5), the additional energy due to a magnetic dipole moment in a magnetic field, is proportional with an in-product of a three dimensional spin vector $\mathbf{S}$, with a quantized amplitude $|\hbar / 2|$, with a three-dimensional magnetic field vector. This allows a simple interpretation of the effect of the electron's angular spin, while the interaction term with the in-product of the Pauli vector with the magnetic field vector in Dirac's wave equation does not immediately allow an energetic interpretation.

3. The third issue that I would like to address is on the composition of the Pauli vector. It is well known that Majorana made a different choice. In this text, I wish to put Dirac's particle in a more general text but electrons. In particular, I wish to model the particle in a general approach by considering different choices for the composition of the Pauli vector.

As just noted, it is rather curious that Dirac's article shows the possible existence of two different dipole moments, while this doesn't show up in most, if not all, textbooks. It might well be that this is related with the general relativity issue. As already stated, from that point of view, one should expect an analysis that includes a four-component Pauli-type vector instead of a three-component one. In fact, Dirac did so, by defining a four-component Paulitype vector $\bar{\alpha}=\bar{\alpha}\left(\alpha_{1}, \alpha_{2}, \alpha_{3}, \alpha_{4}\right)$, functionally related with the Pauli-matrices, but with a higher dimensionality than the Pauli-vector $\overline{\mathrm{O}}=\overline{\mathrm{o}}\left(\mathrm{o}_{1}, \mathrm{O}_{2}, \mathrm{o}_{3}\right)$. However, the full symmetry in his analysis disappeared due to the asymmetry in the metric $(-,+,+,+)$ for space-time $(c t, x, y, z)$. In spite of this, Dirac maintained all features. Dirac could have avoided the seeming asymmetry by using the "Hawking metric" $(+,+,+,+)$ for space-time (ict, $x, y, z)$, which would have considerably simplified his analysis. Nevertheless, the rigor of his analysis showed the arise of two dipole moments, while other texts don't, possibly by not recognizing the subtlety of the metric.

A general approach for covering these issues is rather complicated because of the 4D dimensionality. However, a simplified analysis in 2D might give a lead. Where a 2D analysis cannot provide full knowledge, the results of a proper $4 \mathrm{D}$ analysis should encompass the results of a proper 2D analysis. Hence, it might well be that useful insight can be provided from a $2 \mathrm{D}$ view. The basic elements in such an analysis are a two-dimensional momentum vector $\boldsymbol{p}=\boldsymbol{p}\left(p_{0}, p_{x}\right)$, and a two-dimensional Pauli-vector $\overline{\mathrm{o}}=\overline{\mathrm{o}}\left(\mathrm{o}_{1}, \mathrm{o}_{2}\right)$.

\section{Dirac/Majorana particles in free space}

Let us first consider a generic free moving particle. 
Its Einsteinean energy is given as,

$$
E_{W}=\sqrt{\left(m_{0} c^{2}\right)^{2}+(c|\mathbf{p}|)^{2}}
$$

where $m_{0}$ is the particle's rest mass and where $\mathbf{p}$ is the three-vector momentum $(d s / d t$, not be confused with the fourvector momentum $\boldsymbol{p}$ ). Without loss of generality, the particle's free motion can be aligned along the $x$-axis in a system of Carthesian coordinates, for which we shall adopt the Hawking metric (ict, $x, y, z), \mathrm{i}=\sqrt{-1}$. Squaring (6) gives,

$$
E_{W}^{2}=-p_{0}^{2} c^{2}=\left(m_{0} c^{2}\right)^{2}+c^{2} p_{1}^{2},
$$

which can be normalized as,

$$
p_{0}^{\prime 2}+p_{1}^{\prime 2}+1=0 ; \quad p_{\mu}^{\prime}=\frac{p_{\mu}}{m_{0} c}
$$

Note: As long as the temporal dimension is included, the bold italic notation for the vector $\boldsymbol{p}$ will be maintained.

In retrospect, in Dirac's single mode representation, this equation can be written as,

$$
p_{0}^{\prime 2}+p_{1}^{\prime 2}+1=\left(\beta+\bar{\alpha} \cdot p^{\prime}\right)\left(\beta+\bar{\alpha} \cdot p^{\prime}\right)=0, \text { with } \bar{\alpha}=\bar{\alpha}\left(\alpha_{0}, \alpha_{1}\right) \text { and } \boldsymbol{p}^{\prime}\left(p_{0}^{\prime}, p_{1}^{\prime}\right)
$$

while in Majorana's dual mode representation, this equation can be written as,

$$
p_{0}^{\prime 2}+p_{1}^{\prime 2}+1=\left(\beta+\mathrm{i} \bar{\alpha} \cdot \boldsymbol{p}^{\prime}\right)\left(\beta-\mathrm{i} \bar{\alpha} \cdot \boldsymbol{p}^{\prime}\right)=0
$$

where the components of the two-dimensional vector $\bar{\alpha}=\bar{\alpha}\left(\alpha_{0}, \alpha_{1}\right)$ are given by the Pauli matrices,

$$
\alpha_{0}=\sigma_{1}=\left[\begin{array}{ll}
0 & 1 \\
1 & 0
\end{array}\right], \quad \alpha_{1}=\sigma_{3}=\left[\begin{array}{cc}
1 & 0 \\
0 & -1
\end{array}\right]
$$

and where $\beta$ is a matrix that is slightly different for Dirac's case and Majorana's case, respectively as

$$
\beta=\sigma_{2}=\left[\begin{array}{cc}
0 & -\mathrm{i} \\
\mathrm{i} & 0
\end{array}\right] \text { and } \beta=[I]=\left[\begin{array}{ll}
1 & 0 \\
0 & 1
\end{array}\right] \text {, }
$$

where $[I]$ is the identity matrix.

After transforming the momenta into operators on wave functions, 


$$
p_{\mu}^{\prime} \rightarrow \hat{p}_{\mu} \psi \quad \text { with } \quad \hat{p}_{\mu}^{\prime}=\frac{1}{m_{0} c} \frac{\hbar}{\mathrm{i}} \frac{\partial}{\partial x_{\mu}}
$$

(axiomatic quantum mechanical hypothesis), the momentum relationship (9) is transformed under consideration (11) into the following two two-dimensional wave equations

$$
\left[\alpha_{0}\right]\left[\begin{array}{c}
\hat{p}_{0}^{\prime} \psi_{0} \\
\hat{p}_{0}^{\prime \prime} \psi_{1}
\end{array}\right]+\left[\alpha_{1}\right]\left[\begin{array}{c}
\hat{p}_{1}^{\prime} \psi_{0} \\
\hat{p}_{1}^{\prime} \psi_{1}
\end{array}\right] \pm[\beta]\left[\begin{array}{c}
\psi_{0} \\
\psi_{1}
\end{array}\right]=0 .
$$

The single mode condition is met for a single equation with a + sign in front of $\beta$, while the dual mode falls apart into two simultaneous equations.. This set can be heuristically solved by $\psi\left(\psi_{0}, \psi_{1}\right)$ such that,

$$
\psi_{i}(x, t)=u_{i} \operatorname{expi}\left(\frac{p_{1}}{\hbar} x-\frac{W}{\hbar} t\right)
$$

resulting into,

$$
\begin{aligned}
& \psi_{0}(x, t)=u_{0} \exp \pm \mathrm{i}\left(\frac{p_{1}}{\hbar} x-\frac{W}{\hbar} t\right) ; \quad \psi_{2}(x, t)=u_{2} \exp \pm \mathrm{i}\left(\frac{p_{1}}{\hbar} x-\frac{W}{\hbar} t\right) \\
& \psi_{1}(x, t)=u_{1} \exp \pm \mathrm{i}\left(\frac{p_{1}}{\hbar} x-\frac{W}{\hbar} t\right) ; \quad \text { and } \quad \psi_{3}(x, t)=u_{3} \exp \pm \mathrm{i}\left(\frac{p_{1}}{\hbar} x-\frac{W}{\hbar} t\right) ; \\
& \frac{u_{1}}{u_{0}}=\mp \mathrm{i} \frac{p_{1}}{\left(W / c+m_{0} c\right)} . \quad \frac{u_{2}}{u_{3}}= \pm \mathrm{i} \frac{p_{1}}{\left(W / c+m_{0} c\right)} \text {. }
\end{aligned}
$$

The dual mode allows equating $u_{1}=u_{3}$ and $u_{0}=u_{2}$ and subsequent combining to the wave function set $\psi\left(\psi_{a}, \psi_{b}\right)$, where

$$
\begin{aligned}
& \psi_{a}=u_{a} \cos \left(\frac{p_{1}}{\hbar} x-\frac{W}{\hbar} t\right) \\
& \psi_{b}=u_{b} \sin \left(\frac{p_{1}}{\hbar} x-\frac{W}{\hbar} t\right) ; \\
& \frac{u_{a}}{u_{b}}=\frac{p_{1}}{\left(W / c+m_{0} c\right)}
\end{aligned}
$$

Hence, where the Dirac's single mode solution is a complex wave function, Majorana's dual mode allows a real wave function solution.

\section{The impact of the vector potential on Dirac/Majorana particles in motion}


Let us proceed by considering the impact of a field vector potential $\boldsymbol{A}$ on the free moving particle. To do it properly, an extension of the time-space dimension is required. To avoid the complexity of a full 4D analysis, we shall adopt a second spatial dimension next to the $x$-axis. Hence, we wish to consider now a slightly less simplified system with as basic elements: a " $1+2$ "- dimensional momentum vector $\boldsymbol{p}=\boldsymbol{p}\left(p_{0}, p_{x}, p_{y}\right)$, a three-dimensional Pauli-vector $\overline{\mathrm{O}}=\overline{\mathrm{O}}\left(\mathrm{O}_{1}, \mathrm{O}_{2}, \sigma_{3}\right)$ and a three-dimensional vector potential $\boldsymbol{A}\left(A_{0}, A_{x}, A_{y}\right)$. In these terms, the free-space Einsteinean energy (9) is expressed as

$$
[I]+\left(\bar{\sigma} \cdot p^{\prime}\right)\left(\bar{\sigma} \cdot p^{\prime}\right)=0
$$

A basic property of the Pauli-vector says,

$$
(\bar{\sigma} \cdot \mathbf{v})(\cdot \overline{\bar{\alpha}} \cdot \mathbf{w})=[I](\mathbf{v} \cdot \mathbf{w})+\mathrm{i}(\mathbf{v} \times \mathbf{w}) \cdot \bar{\sigma},
$$

where $\mathbf{v}$ and $\mathbf{w}$ are two generic three-component vectors.

Applying (16) in (15) yields two possible results,

$$
(\bar{\sigma} \cdot \mathbf{v})(\cdot \bar{\sigma} \cdot \mathbf{w})=\mathbf{v} \cdot \mathbf{w} \pm|\mathbf{v} \times \mathbf{w}|
$$

Hence, from (15) and (17)

$$
[I]+\left(\bar{\sigma} \cdot \boldsymbol{p}^{\prime}\right)\left(\bar{\sigma} \cdot \boldsymbol{p}^{\prime}\right)=0 \rightarrow 1+\boldsymbol{p}^{\prime} \cdot \boldsymbol{p}^{\prime} \pm\left|\boldsymbol{p}^{\prime} \times \boldsymbol{p}^{\prime}=0\right|
$$

This might seem a trivial result, because the vector product of a vector with itself is zero. Hence, this is just a retrieval of the Einsteinean energy expression (8). However, under influence of the presence of a conservative field of forces, characterized by a (normalized) vector potential $A^{\prime}$, the expression changes under the change of momenta components,

$$
\boldsymbol{p}^{\prime} \rightarrow \boldsymbol{p}^{\prime}+A^{\prime}
$$

such that (18) transforms to,

$$
1+\boldsymbol{p}^{\prime} \cdot \boldsymbol{p}^{\prime} \pm\left|\boldsymbol{p}^{\prime} \times \boldsymbol{p}^{\prime}\right|=0 \rightarrow 1+\left(\boldsymbol{p}^{\prime}+A^{\prime}\right) \cdot\left(\boldsymbol{p}^{\prime}+A^{\prime}\right) \pm\left|\left(\boldsymbol{p}^{\prime}+A^{\prime}\right) \times\left(\boldsymbol{p}^{\prime}+A^{\prime}\right)\right|=0
$$

The vector product in this expression still seems being irrelevant, because of its zero value. This, however, changes after the quantum mechanical transform from momenta to operations on a wave function, defined by

$$
p_{\mu}^{\prime} \rightarrow \hat{p}_{\mu} \psi \quad \text { with } \quad \hat{p}_{\mu}^{\prime}=\frac{1}{m_{0} c} \frac{\hbar}{\mathrm{i}} \frac{\partial}{\partial x_{\mu}}
$$

Applying these transforms on the generic identity 
$(\mathbf{v}+\mathbf{w}) \times(\mathbf{v}+\mathbf{w})=(\mathbf{v} \times \mathbf{x})+(\mathbf{w} \times \mathbf{v})$

we have

$$
\left(p^{\prime}+A^{\prime}\right) \times\left(p^{\prime}+A^{\prime}\right) \rightarrow\left(\hat{p}^{\prime} \times A^{\prime}\right) \psi+\left(A^{\prime} \times \hat{p}^{\prime}\right) \psi
$$

Where the operator in the first term operates on $\psi$ as well as on $\boldsymbol{A}^{\prime}$, the operator in the second term only operates on $\psi$. As a consequence (22) is evaluated as,

$$
\left(\boldsymbol{p}^{\prime} \times \boldsymbol{A}^{\prime}\right)+\left(\boldsymbol{A}^{\prime} \times \boldsymbol{p}^{\prime}\right)=\frac{\hbar}{\mathrm{i} m_{0} c} \psi\left(\nabla \times A^{\prime}\right) .
$$

Where the vector product of the momentum representation is zero, the equivalent wave function representation is not. Apparently, the Einsteinean energy of a particle moving with a momentum $\boldsymbol{p}=\boldsymbol{p}\left(p_{0}, p_{x}, p_{y}\right)$ under influence of a three-dimensional vector potential $\boldsymbol{A}\left(A_{0}, A_{x}, A_{y}\right)$ is given by,

$$
\left(\boldsymbol{p}^{\prime}+\boldsymbol{A}^{\prime}\right)\left(\boldsymbol{p}^{\prime}+\boldsymbol{A}^{\prime}\right)+1 \pm\left|\frac{\hbar}{\mathrm{i} m_{0} c}\left(\nabla \times \boldsymbol{A}^{\prime}\right)\right|=0
$$

The last term in the right hand part of this expression represents the energy added by the interaction of the particle's spin with the vector potential.

If we would have considered a 4D dimensionality in our analysis instead of the simplified " 1 +2 " one, we would have met the difficulty to generalize the curl operation, which shows up in (24), to a 4D equivalent. In many texts on the subject, the problem is avoided by giving up symmetry and separating the temporal momentum from the spatial momenta, thereby excluding the temporal momentum from the curl operation. This, for instance, can be seen in Bjorken and Drell's textbook by moving from [4, eq. 1.32) to (4, eq.1.34). Here, the temporal momentum is not included in the curl operation. As a consequence only a single dipole moment shows up, while in Dirac's comparable expression [1, eq. 15/16] two dipole moments are shown, albeit that Dirac could not maintain full symmetry in his representation because of the asymmetry in the metric $(-,+,+,+)$. At this point, it is interesting to note that Lanczos [7] has been able to maintain full symmetry in the curl operation owing to his special quaternion algebra, which enabled him to give an interpretation to "isospin" and that Hestenes [8,9] developed his special STA algebra for the purpose, which enabled to explain the "zitterbewegung" of electrons. Dirac's second dipole moment, Lanczos' isospin and Hestenes' zitterbewegung are related and can be traced back to the inclusion of the temporal moment in the curl operation that shows up as a consequence of the interaction of the Pauli vector with the vector potential. The simplification from 4D space-time to " $1+2$ " space-time may have its limitations, but, on the other hand, it enables maintaining full symmetry under the use of the "Hawking" metric $(+,+,+,+)$ for space-time (ict, $x, y, z)$.

Generically, the vector potential $\boldsymbol{A}$ consists of a scalar component $\Phi$ next to a vector component $\mathbf{A}$. If the particle would be spin less, a free motion along the $x$-axis would be 
possible under the equilibrium of a force from a transversal component of the scalar field $\Phi$ and the Lorentz force from a orthogonal component $B_{z}$ created from $\nabla \times \mathbf{A}$, i.e. from components $A_{x}$ and $A_{y}$. The interaction of the vector potential with the Pauli spin vector, however disturbs such an equilibrium. Let us consider the energetic influence of the interaction. Let the momentum vector and the vector potential, respectively be given by,

$\boldsymbol{p}^{\prime}=\boldsymbol{p}^{\prime}\left(p_{0}^{\prime}, p_{x}\right)$ and $\boldsymbol{A}^{\prime}=\boldsymbol{A}^{\prime}\left(\mathrm{i} \frac{\Phi / c}{m_{0} c}, A_{x}^{\prime}, A_{y}^{\prime}\right)$

Note: The i factor in the scalar component is due to the (Hawking) metric choice $(+,+,+,+) /$ (ict, $x, y, z)$. It can be easily seen from the Lorenz gauge

$$
\nabla \cdot \mathbf{A}+\frac{1}{c^{2}} \frac{\partial \Phi}{\partial t}=0 \rightarrow \nabla \cdot \mathbf{A}+\mathrm{i} \frac{\partial \Phi / c}{\partial \mathrm{i} c t}=0
$$

Note also that $\Phi / m_{0} c^{2}$ is a dimensionless quantity. Under the conditions of stationarity of $\boldsymbol{A}$ and exclusive dependence of $\Phi$ on $y$, we have, under consideration of (25)

$\nabla \times \boldsymbol{A}^{\prime}=\left[\begin{array}{ccc}\mathbf{e}_{\mathbf{t}} & \mathbf{e}_{\mathbf{x}} & \mathbf{e}_{\mathbf{y}} \\ \partial / \mathrm{i} c t & \partial / \partial x & \partial / \partial y \\ \mathrm{i} \Phi / m_{0} c^{2} & A_{x}^{\prime} & A_{y}^{\prime}\end{array}\right]=-\frac{\partial}{\partial y} \mathrm{i} \frac{\Phi}{m_{0} c^{2}} \mathbf{e}_{\mathbf{x}}+\left(\frac{\partial}{\partial x} A_{y}^{\prime}-\frac{\partial}{\partial y} A_{x}^{\prime}\right) \mathbf{e}_{\mathbf{t}}$

where $\mathbf{e}_{\mathbf{x}}, \mathbf{e}_{\mathbf{y}}$ and $\mathbf{e}_{\mathbf{t}}$, respectively, are unit vectors along the two spatial axes and the temporal axis.

Because of $\mathbf{B}=\nabla \times \mathbf{A}$, we have

$\frac{\partial}{\partial x} A_{y}^{\prime}-\frac{\partial}{\partial y} A_{x}^{\prime}=B_{z}^{\prime}$

Because $\mathbf{e}_{\mathbf{t}}$ is a unit vector along the imaginary axis, both contributions in (27) are imaginary, thereby making a real contribution in (24). Hence, the additional energy $\Delta E$ due to the interaction of the Pauli vector with the vector potential amounts to

$$
\Delta E=\left|\frac{\hbar}{m_{0} c} \frac{\partial}{\partial y} \frac{\Phi}{m_{0} c^{2}}+\frac{\hbar}{2 c} \frac{B_{z}}{m_{0} c}\right|
$$

Adding this amount to the energy of the moving particle, we have

$$
E_{W}^{\prime 2}=p_{1}^{\prime 2}+1 \pm\left|\frac{\hbar}{m_{0} c} \frac{\partial}{\partial y} \frac{\Phi}{m_{0} c^{2}}+\frac{\hbar}{2 c} \frac{B_{z}}{m_{0} c}\right|
$$

After denormalization, 


$$
\left(\frac{E_{W}}{m_{0} c^{2}}\right)^{2}=\left(\frac{m_{0} v_{x}}{m_{0} c}\right)^{2} \pm\left|\frac{\hbar}{m_{0} c} \frac{\partial}{\partial y} \frac{\Phi}{m_{0} c^{2}}+\frac{\hbar}{2 c} \frac{B_{z}}{m_{0} c}\right|+1=0 .
$$

Supposing that the term between brackets is much smaller than 1 ,

$$
\begin{aligned}
& E_{W} \approx\left(m_{0} c^{2}\right)\left(1+\frac{1}{2} \frac{v^{2}}{c^{2}} \pm\left|\frac{\hbar}{m_{0} c} \frac{\partial}{\partial y} \frac{\Phi}{m_{0} c^{2}}+\frac{\hbar}{2 c} \frac{B_{z}}{m_{0} c}\right|\right)= \\
& \left.=m_{0} c^{2}\right)\left(1+\frac{1}{2} \frac{v^{2}}{c^{2}}\right) \pm\left(\frac{\hbar}{2 c} \frac{1}{m_{0}} \frac{\partial}{\partial y} \Phi+\frac{\hbar}{2} \frac{B_{z}}{m_{0}}\right) .
\end{aligned}
$$

So far, the Dirac particle has been considered in general terms, i.e. without identifying it as an electron. To do so, the potential $\Phi$ has to be interpreted by the force equity $F$ as,

$$
F=q \frac{\partial}{\partial y} \Phi_{\mathrm{e}}=\frac{\partial}{\partial y} \Phi \rightarrow \Phi_{\mathrm{e}}=\frac{\Phi}{q} ; B_{z}^{e m}=\frac{B_{Z}}{q}
$$

where $q$ is the electric charge of an electrical particle under consideration and where $\Phi_{\mathrm{e}}$ and $B_{z}^{e m}$, respectively, are the electric potential and the magnetic field. Hence, from (32) and (33),

$$
\left.E_{W}=m_{0} c^{2}\right)\left(1+\frac{1}{2} \frac{v^{2}}{c^{2}}\right) \pm\left(\frac{\hbar}{2 c} \frac{q}{m_{0}} \frac{\partial}{\partial y} \Phi_{\mathrm{e}}+\frac{\hbar q}{2 m_{0}} B_{z}^{e m}\right) .
$$

From (34) the influence of the Pauli spin on the motion of the particle under influence of the balance between the force of a transversal electric field and the Lorentz force from a magnetic field perpendicular on the motion as well as on the electric field, becomes clear from the last two terms in the right-hand part (34). Here, two dipole moments are identified. Apart from the well-known dipole moment $\hbar q / 2 m_{0}$ operating on the magnetic field, as mentioned before in (3), there appears a second dipole moment $\hbar q / 2 \mathrm{~cm}_{0}$ operating on the electric field, as mentioned before in (4).

\section{Comparison with Dirac's analysis}

Similar as in Dirac's analysis two dipole moments show up. However, where Dirac concluded that the electric dipole moment is not feasible, because of the imaginary term that shows up after evaluation of [1, eq. (15)], the electric dipole moment in (34) shows up as real. Let us try to understand what the origin could be of the difference. To do so, let us closely inspect Dirac's [1,eq.(15)]. His Hamiltonian contains the term

$\operatorname{Trm}=\rho_{1}\left[\left\{\bar{\sigma} \cdot\left(\hat{\mathbf{p}}^{\prime}+q \mathbf{A}^{\prime}\right)\right\}\left(\hat{p}_{0}^{\prime}+q A_{0}^{\prime}\right)-\left(\hat{p}_{0}^{\prime}+q A_{0}^{\prime}\right)\left\{\bar{\sigma} \cdot\left(\hat{\mathbf{p}}^{\prime}+q \mathbf{A}^{\prime}\right)\right\}\right.$, 
where $\rho_{1}$ is a $4 \times 4$ matrix derived from the $2 \times 2$ identity matrix to account for the four modalities of Dirac's equation.

Let us simplify this term by omitting all time dependencies. Hence

Term $=\rho_{1}\left[\left\{\bar{\sigma} \cdot\left(\hat{\mathbf{p}}^{\prime}+q \mathbf{A}^{\prime}\right)\right\} q A_{0}^{\prime}-q A_{0}^{\prime}\left\{\bar{\sigma} \cdot\left(\hat{\mathbf{p}}^{\prime}+q \mathbf{A}\right)\right]\right.$

(Note that, in Dirac's analysis $A_{0}$ is real as $A_{0}=\Phi_{\mathrm{e}}$ ).

Let us now skip the terms that obviously cancel. This gives,

$\operatorname{Term}=\rho_{1}\left[\left(\bar{\sigma} \cdot \hat{\mathbf{p}}^{\prime}\right) q A_{0}^{\prime}-q A_{0}^{\prime}\left(\bar{\sigma} \cdot \hat{\mathbf{p}}^{\prime}\right)\right]$

This term evolves as,

Term $=\mathrm{i} q \rho_{1} \hbar \bar{\sigma} \cdot\left(\frac{\partial A_{0}^{\prime}}{\partial x} \mathbf{i}+\frac{\partial A_{0}^{\prime}}{\partial y} \mathbf{j}+\frac{\partial A_{0}^{\prime}}{\partial z} \mathbf{k}\right)$

Expanding this term gives

Term $=\mathrm{i} q \hbar \rho_{1}\left(\cdot \sigma_{1} \mathbf{i}+\sigma_{2} \mathbf{j}+\sigma_{3} \mathbf{k}\right) \cdot\left(\frac{\partial A_{0}^{\prime}}{\partial x} \mathbf{i}+\frac{\partial A_{0}^{\prime}}{\partial y} \mathbf{j}+\frac{\partial A_{0}^{\prime}}{\partial z} \mathbf{k}\right)$

Let us simplify even more by considering the same condition as in our " $1+2$ " approach. This gives

Term $=\mathrm{i} q \hbar \rho_{1}\left[\begin{array}{cc}0 & -\mathrm{i} \\ \mathrm{i} & 0\end{array}\right] \frac{\partial A_{0}^{\prime}}{\partial y}=\frac{\mathrm{i} q \hbar \rho_{1}}{m_{0} c}\left[\begin{array}{cc}0 & -\mathrm{i} \\ \mathrm{i} & 0\end{array}\right] \frac{\partial \Phi_{\mathrm{e}}}{\partial y}$.

Hence, in spite of Dirac's opinion, Term shows different modes that reveal a real electric dipole moment. Hence, after all, the conclusion drawn from the simplified analysis in the "1 +2 " domain is not in conflict with Dirac's generic result as shown in [1,eq.(15)]. However, Dirac's interpretation that the electric dipole moment is imaginary seems doubtful.

\section{Discussion}

At first glance, it might seem that the impact of the conclusion that Dirac's second dipole moment is real, is of limited value. The view on its possible impact might change if we put a Dirac/Majorana particle in a more general context. Let us suppose that a quark can be conceived as a Dirac/Majorana particle as well. In such a scope, we may assign a nuclear field $\Phi_{\mathrm{qu}}$ to a quark and, in addition, we may suppose that an antiquark may couple to this field by a coupling factor $g$ (from the electromagnetic fine constant expression $q^{2}=4 \pi \varepsilon_{0} \hbar c g^{2}$ ) such that, 


$$
F=g \frac{\partial}{\partial y} \Phi_{\mathrm{qu}}=\frac{\partial}{\partial y} \Phi \rightarrow \Phi_{\mathrm{qu}}=\frac{\Phi}{g}
$$

In a recent preprint [10], I have demonstrated the consequences of such a model, thereby revealing that it gives an adequate explanation for the mass spectrum of leptons and hadrons. Because the second dipole moment of the quark is shown as being the origin of isospin as well as being the gluing force between the quarks, such an approach is considered being in conflict with the QCD color force binding by gluons. Hence, this theory meets fierce opposition, in spite of the unprecedented successful calculation of mass values with an undeniable match with experimental evidence. Some years ago, before being aware of the second dipole moment I have used the very same model for successfully expressing Newton's gravitational constant into quantum mechanical quantities [11]. Another generalization of Dirac/Majorana particles is conceivable in the gravitational domain with gravitational potential $\Phi_{\mathrm{G}}$.

$$
F=m_{0} \frac{\partial}{\partial y} \Phi_{\mathrm{G}}=\frac{\partial}{\partial y} \Phi \rightarrow \Phi_{\mathrm{G}}=\frac{\Phi}{m_{0}}
$$

In attempts to give an explanation for cosmological phenomena as dark matter and dark energy, the universe is modeled as a tenuous gaseous medium with background energy [12]. By modeling the gaseous molecules as Dirac/Majorana particles, these cosmological phenomena can be explained as a consequence of vacuum polarization and verified by observational evidence [13].

Although these studies have led the author to the rediscovery of Dirac's second dipole moment, a decisive experimental proof would be most welcome. How to construct such a proof is not obvious. The phenomenon to be shown and measured is the second spin-flip of an electron under influence of a vector potential. A first option is the use of electron spin resonance spectrography (ESR), [14]. Unfortunately, the spectral split due to the anomalous electric dipole moment is masked by the electron's orbital motion in atoms, because the orbital motion cancels symmetric contributions. As a second option one might consider the hyperfine split effect due to the spin-spin interaction of the electron with the atomic nucleus, which gives rise to the well-known $21 \mathrm{~cm}$ line in the cosmological electromagnetic spectrum of atomic hydrogen [15]. Unfortunately, the interaction energy between the spins due to the anomalous electric dipole moments is just equal to the interaction energy due to the anomalous magnetic dipole moments. This can be seen as follows. According to Griffiths $[5,16]$, the interaction energy $\Delta E$ between the magnetic dipoles $\mu_{m}^{e}$ and $\mu_{m}^{p}$ of, respectively, the electron (mass $m_{e}$ ) and the proton (mass $m_{p} ;$ " $\mathrm{g}$ "-factor $g_{p}=5.59$ ) amounts to

$$
\Delta E=\mu_{0} \frac{g_{p} \mu_{m}^{e} \mu_{m}^{p}}{3 \pi a_{0}^{3}}=\frac{4 g_{p} \hbar^{4}}{3 a_{0}^{4} m_{p} m_{e}^{2} c^{2}} ; \quad a_{0}=\frac{\hbar}{g^{2} m_{e} c}
$$

where $a_{0}$ is the Bohr radius[17].

Hence, from 
$\Delta E=\hbar \omega=h f \rightarrow f \approx 1.42 \mathrm{GHz} \rightarrow \lambda \approx 21 \mathrm{~cm}$.

The interaction energy $\Delta E_{e l}$ between the electric dipoles $\mu_{e l}^{e}$ and $\mu_{e l}^{p}$ of, respectively, the electron and the proton amounts to

$\Delta E_{e l}=\varepsilon_{0} \frac{g_{p} \mu_{e l}^{e} \mu_{e l}^{p}}{3 \pi a_{0}^{3}}$

Because of the relationship between the magnetic dipoles and electric dipoles as expressed by (3) and (4) and because $c^{2}=\left(\varepsilon_{0} \mu_{0}\right)^{-1}$, the two interaction energies $\Delta E_{e l}$ and $\Delta E$ are just the same.

In view of this, a proof for the existence of an electric dipole moment of electrons is far from easy. It might even be a reason to deny its relevancy, like Dirac did. This might be different for quarks, if one is willing to consider the hypothesis that a quark is a Dirac particle as well, albeit of a different kind. Let the quark be conceived as a pointlike source with an elementary angular momentum $\hbar / 2$ and an elementary linear momentum $\hbar / 2 c$ and let this quark spread a field $\Phi(r)=\Phi_{F}(r)+\Phi_{N}(r)$, with a far field component $\Phi_{F}(r)$ and a near field component $\Phi_{N}(r)$. As can be expected, the most simple expression for the (near) field from the linear momentum will show up along the axis $x$ set up between the poles. Under consideration of a generic dipole moment $m_{p} d$, this potential field can be readily derived as,

$\frac{\Phi_{N}(x)}{m_{0}}=\frac{G m_{p} d}{x^{2}} \rightarrow \frac{\Phi_{N}(x)}{m_{0}}=\frac{\hbar}{2 c} \frac{\lambda^{2} G}{(\lambda x)^{2}} \rightarrow \frac{\Phi_{N}(x)}{m_{0}}=\frac{\Phi_{0}}{m_{0}} \frac{1}{(\lambda x)^{2}} ; \quad \frac{\Phi_{0}}{m_{0}}=\frac{\hbar}{2 c} G \lambda^{2}$.

Whether the proportionality constant $G$ is the Newtonian one or not, is not relevant for the present discussion. What matters is the generic expression in terms of a strength parameter $\Phi_{0}$ and a spatial parameter $\lambda$. Note that the mass $m_{p}$ of the fictitious pole in the elementary dipole is different from the effective mass $m_{0}$ in the massive energy of the Dirac particle. Because it is just an auxiliary quantity, the relevancy of mass $m_{p}$ is restricted.

The far field is the result of an effective mass from the elementary angular moment. Interpreting the angular momentum as a rotation with light speed at a fictitious radius $r_{0}=1 / g_{m} \lambda$, we have

$\frac{\hbar}{2}=\frac{m_{p} c}{g_{m \lambda} \lambda} \rightarrow m_{p}=g_{m} \frac{\hbar}{2} \frac{\lambda}{c}$

The quantity $g_{m}$ is an unknown gyrometric constant.

Hence, from classical field theory, 
$\frac{\Phi_{F}(r)}{m_{0}}=\frac{m_{p} G}{r}=g_{m} \frac{\hbar}{2} \frac{\lambda}{c} \frac{G}{r}=g_{m} \frac{\hbar}{2} \frac{G \lambda^{2}}{c} \frac{1}{\lambda r}$

and, under consideration of $\Phi_{0}$ as defined in (46),

$\frac{\Phi_{F}(r)}{m_{0}}=g_{m} \frac{\hbar}{2} \frac{G \lambda^{2}}{c} \frac{1}{\lambda r}=\frac{\Phi_{0}}{m_{0}} \frac{g_{m}}{(\lambda r)}$

These two potential fields, $\Phi_{N}(x)$ in (46) and $\Phi_{F}(r)$ in (49), are the results of the energetic flow from the Dirac particle-type source. It can be influenced by a background (Higgs) field. Assuming such influence and assuming that the near field is attracting and that the far field is repulsive, the quark's potential field along the axis set up between the poles of the linear momentum has the format,

$\Phi(\lambda x)=\Phi_{0} \exp (-\lambda x)\left\{\frac{1}{(\lambda x)^{2}}-g_{m} \frac{1}{\lambda x}\right\}$

The potential field has the well known liquid drop format, known from the potential field between nucleons. Each of two quarks in a meson are coupled to the field of the other with the generic quantum mechanical coupling factor $g$. Because of the electroweak hypothesis this coupling factor is taken as the square root from the electromagnetic fine structure constant $g^{2}$, as shown in (41). Hence, the combined field from two quarks in a meson aligned along the $x$-axis, spaced at a $2 d$ distance, can be expanded as,

$V(x)=\Phi(d+x)+\Phi(d-x)=g \Phi_{0}\left(k_{0}+k_{2} \lambda^{2} x^{2}+\ldots \ldots\right)$,

where $k_{0}$ and $k_{2}$ are dimensionless coefficients with magnitudes that depend on the spacing $d$. The interesting feature of the meson configuration now is, that its center of mass is subject to a potential that (almost) depends on the square of $x$. The two quarks in the meson align themselves in the condition of minimum energy at $d \lambda=d_{\min }^{\prime}$. Hence, the meson shows the characteristics of a quantum mechanical oscillator. It is therefore subject to excitation, which is the underlying mechanism for the systematic characteristic of the mass spectrum of mesons [18].

The intriguing conclusion therefore is that Dirac's second dipole moment may give a physical interpretation to the gluing force between quarks and antiquark, which in the Standard Model of particle physics is axiomatically conceived from a mathematical framework as the QCD color force. It is also obvious that an another axiomatic attribute in the Standard Model, namely the isospin, gets a physical interpretation as well. This does not necessarily imply that the two approaches are necessarily incompatible. They might be two sides of the same medal.

\section{Conclusion}


A Dirac/Majorana particle has two anomalous dipole moments. One of these is the consequence of an elementary angular moment assigned to the pointlike particle. For electrons It becomes manifest as a magnetic dipole moment. The second one comes forward as the result of Dirac's modeling, but remained forgotten because of a number of reasons. The main one is Dirac's perception that it has no physical relevance as an electric dipole moment, because of its seeming imaginary value. A second one is its disappearance in Diractype analyses in standard textbooks. A third reason is the failure of proof by measurements. A fourth reason is the perceived violation with the quantum mechanical CPT theorem. In this article, first of all a proof is given for the inconsistency between Dirac's result on the dipole moments and the Bjorken and Drell textbook result. The reason why has been shown. By simplifying the analysis to a domain with, next to the temporal axis, only two spatial axes and comparing the result with the result from Dirac's analysis, it could be shown that Dirac's conclusion on the perceived imaginary value of the second dipole moment is doubtful, if not incorrect. Subsequently, the remaining issues of failure of experimental evidence and CPT violation have been removed by argumentation. Moreover, it has been argued that the Heisenberg vibration uncertainty, as an elementary motion next to the angular one, allows a physical interpretation Finally, from referenced literature, it has been indicated that beyond the domain of electrons, Dirac's second dipole moment may have a significant impact in the nuclear domain and the gravitational domain.

\section{References}

[1] P.A.M. Dirac, Proc.Royal Soc. London, A 117, 610 (1928)

[2] M. Tanabashi et al. (Particle Data Group), Phys. Rev. D 98, 030001 (2018)

[3] Y.K. Semertzidis, Nuclear Phys. B, 131, 244 (2004)

[4] J.D. Bjorken, S.D. Drell, Relativistic Quantum Mechanics, McGraw-Hill Book Cie (1964)

[5] D. Griffiths, Introduction to Elementary Particles, ISBN 3527406018, Wiley (2008)

[6] L.I. Mandelshtam, I.E. Tamm, J. Phys (USSR) 9, 249 (1945)

[7] C. Lanczos, F. Phys, 57, 447 (1929), reprint by W.R. Davis et al., North Car. St. Univ., Raleigh, Vol III, p2-1248 (1988)

[8] D. Hestenes, Found Phys. 40, doi.org/10.1007/s10701-009-9360-3 (2010)

[9] D. Hestenes, ArXiv: 0.802.2728v1 (2008)

[10] E. Roza, doi: 10.20944/preprints201905.0118.v1(2019)

[11] E. Roza, Results in Physics, 6 DOI: 10.1016/j.rnp 2016.03.001 (2016)

[12] T. Jacobson, Phys.Rev. Lett., 116, no. 20, 201101 (2016)

[13] E. Roza, Astrophys. And Space Sci., 364:73, doi.org/10.1007/s10509-019-3561-9 (2019)

[14] https://en.wikipedia.org/wiki/Electron_paramagnetic_resonance

[15] C.A. Muller, J.H. Oort, Nature 168 (4270), 357 (1951)

[16] https://physicspages.com/pdf/Griffiths QM/ Griffiths Problems 06.27.pdf

[17] "CODATA Value Bohr radius" Fund. Phys. Constants, NIST, retrieved Feb. 2016

[18] E. Roza, preprints.org, doi: 10.20944/preprints201701.0076.v1 (2017) 